\title{
Late Device Embolization after MitraClip Procedure for Degenerative Mitral Regurgitation
}

\author{
Nobuyuki Fukuda, Hiroshi Ueno, Shuhei Tanaka, Ryuichi Ushijima, Mitsuo Sobajima, Shigeki Yokoyama, Toshio Doi,
} Kazuaki Fukahara, and Koichiro Kinugawa

The Second Department of Internal Medicine, University of Toyama, Toyama University Hospital Cardiovascular Center, Toyama, Toyama, Japan

Received: October 4, 2019; Accepted: March 23, 2020

Objective: MitraClip is currently indicated for symptomatic severe mitral regurgitation (MR) in surgical high-risk patients. After the MitraClip procedure, single leaflet device attachment and device embolization appear as potential risks that have yet to be clarified. We present a case with occult single leaflet device attachment followed by late device embolization.

Case Presentation: A 60-year-old man presented with severe symptomatic MR due to wide prolapse of the posterior leaflet, resembling Barlow syndrome. Medical history included pancreatic cancer (Stage IVb) at 52 years old, and lung metastases at 59 years old. He became aware of heart failure symptoms due to MR and surgery was indicated. After a multidisciplinary heart team conference, the decision was made to perform the MitraClip procedure. During the MitraClip procedure, attempted placement of the second clip interfered with the chorda tendineae, control of the clip was lost and both clips became entangled. Eventually, two clips were placed, and MR improved to a mild degree, but stability of the first clip was insufficient. Five months later, the first clip became detached from the posterior leaflet. At 6 months after the procedure, the clip had dropped to the inferior wall of the left ventricular. We diagnosed clip embolization of the first clip, performed emergency open heart surgery.

Conclusion: Clip instability may impose a higher risk of clip embolization in the MitraClip procedure. We recognized not only reduction of MR but also clip stability as important factors to determine success of the MitraClip procedure, particularly with degenerative MR.

Keywords: MitraClip, degenerative mitral regurgitation, late embolization, single leaflet device attachment

\section{Introduction}

Use of the MitraClip is currently indicated for high-risk patients with severe symptomatic mitral valve regurgitation. ${ }^{1,2)}$ Primary mitral regurgitation (MR) is usually due to an organic cause, whereas secondary MR is due to secondary ischemia or cardiomyopathy. The MitraClip

Corresponding author: Nobuyuki Fukuda. The Second Department of Internal Medicine, University of Toyama, 2630 Sugitani, Toyama, Toyama 930-0194, Japan

Email:nfukuda@med.u-toyama.ac.jp

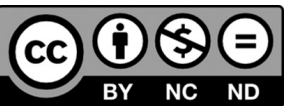

This work is licensed under a Creative Commons Attribution-NonCommercialNoDerivatives International License.

(C)2020 Japan Transcatheter Valve Therapies procedure has been reported as safe and effective among patients with heart failure and severe MR. ${ }^{3)}$ Although the MitraClip procedure can be considered safe with few associated complications, device-related complications such as single leaflet device attachment, device embolization, and endocarditis have been recognized. ${ }^{4-9)}$ We here report a case with late embolization of a clip to the left ventricle in a patient with heart failure and degenerative severe MR, who was at surgical risk of metastatic malignancy.

\section{Case Report}

A 60-year-old man presented with severe symptomatic MR due to wide posterior leaflet prolapse and elongation 

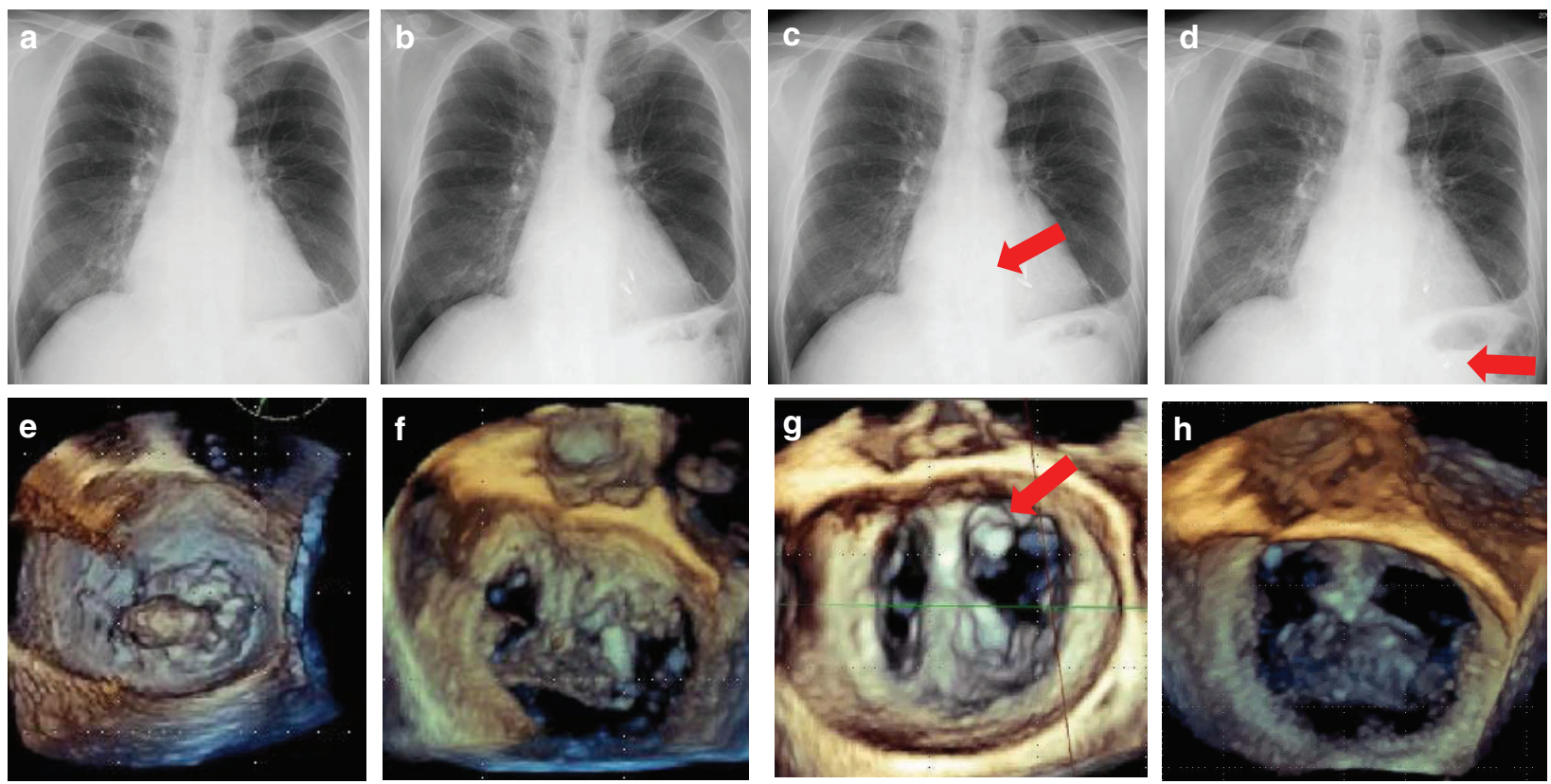

Fig. 1 Chest X-rays (upper row) and images of the mitral valve from three-dimensional transesophageal echocardiography (lower row). a, e: Before MitraClip procedure. b, f: Immediately after MitraClip deployment. c, g: 5 months after MitraClip deployment (single leaflet device attachment). d, h: 6 months after MitraClip deployment (late clip embolization). Red arrows indicate clips.

of the chordae tendineae, resembling Barlow syndrome. Severe MR had been diagnosed by transthoracic echocardiography since he was 52 years old at a time when he received resection of stage $\mathrm{IVb}$ pancreatic cancer. At that time, the surgery was completed without cardiac events. Atrial fibrillation and left ventricular dilatation were observed at 59 years old. Lung metastases from the pancreatic cancer were also found at 59 years old. Since then, he had received anticancer drug treatment, which proved effective and stage of cancer was stable. However, he became aware of dyspnea under light exertion. He received medical treatment for heart failure, but exertional dyspnea gradually worsened. He was referred to our cardiovascular center for evaluation and treatment of MR at 60 years old.

On presentation, he was taking medications for heart failure such as diuretics, angiotensin II receptor blocker, beta-blockers, and anticoagulants, and was taking S-1 as an anticancer drug. A third heart sound and holosystolic murmur were heard at the apex. Plasma B-type natriuretic peptide level reached as high as $191 \mathrm{pg} / \mathrm{mL}$. Transthoracic echocardiography showed an enlarged left atrium and left ventricle, but preserved left ventricular systolic function. Left ventricular diastolic dimension was $71 \mathrm{~mm}$ and ejection fraction was $63 \%$ on transthoracic echocardiography. Severe MR due to extensive, broad prolapse of the posterior mitral leaflet was confirmed. The effective regurgitant orifice area was $0.65 \mathrm{~cm}^{2}$ and regurgitant volume was $68 \mathrm{~mL}$. Transesophageal echocardiography revealed extensive prolapse of the posterior leaflet and elongation of the chordae tendineae, like Barlow syndrome, with myxomatous changes. Figure 1 (bottom row, e) shows mitral morphology on threedimensional echocardiography. Mitral morphology was examined by two-dimensional and three-dimensional echocardiography. According to the German consensus, ${ }^{10)}$ mitral valve area $\left(5.1 \mathrm{~cm}^{2}\right)$ met the criteria for optimal morphology for MitraClip application, but flail gap $(10 \mathrm{~mm})$ and flail width $(20 \mathrm{~mm})$ suggested that the MitraClip procedure was not indicated. Figure 2 shows the mitral morphology on two-dimensional echocardiography. Our multidisciplinary heart team discussed this point. According to the present guidelines, ${ }^{2}$ patients with symptomatic degenerative MR and preserved left ventricular ejection fraction are considered as the class I indication for surgical procedures. In the EVEREST II trial, surgical mitral valve replacement or valvuloplasty was favorable for younger patients with degenerative MR with preserved left ventricular ejection fraction who can withstand open heart surgery. ${ }^{6,7)}$ However, in cases with advanced malignancy, the use of cardiopulmonary bypass may cause transient immunosuppression and thus 

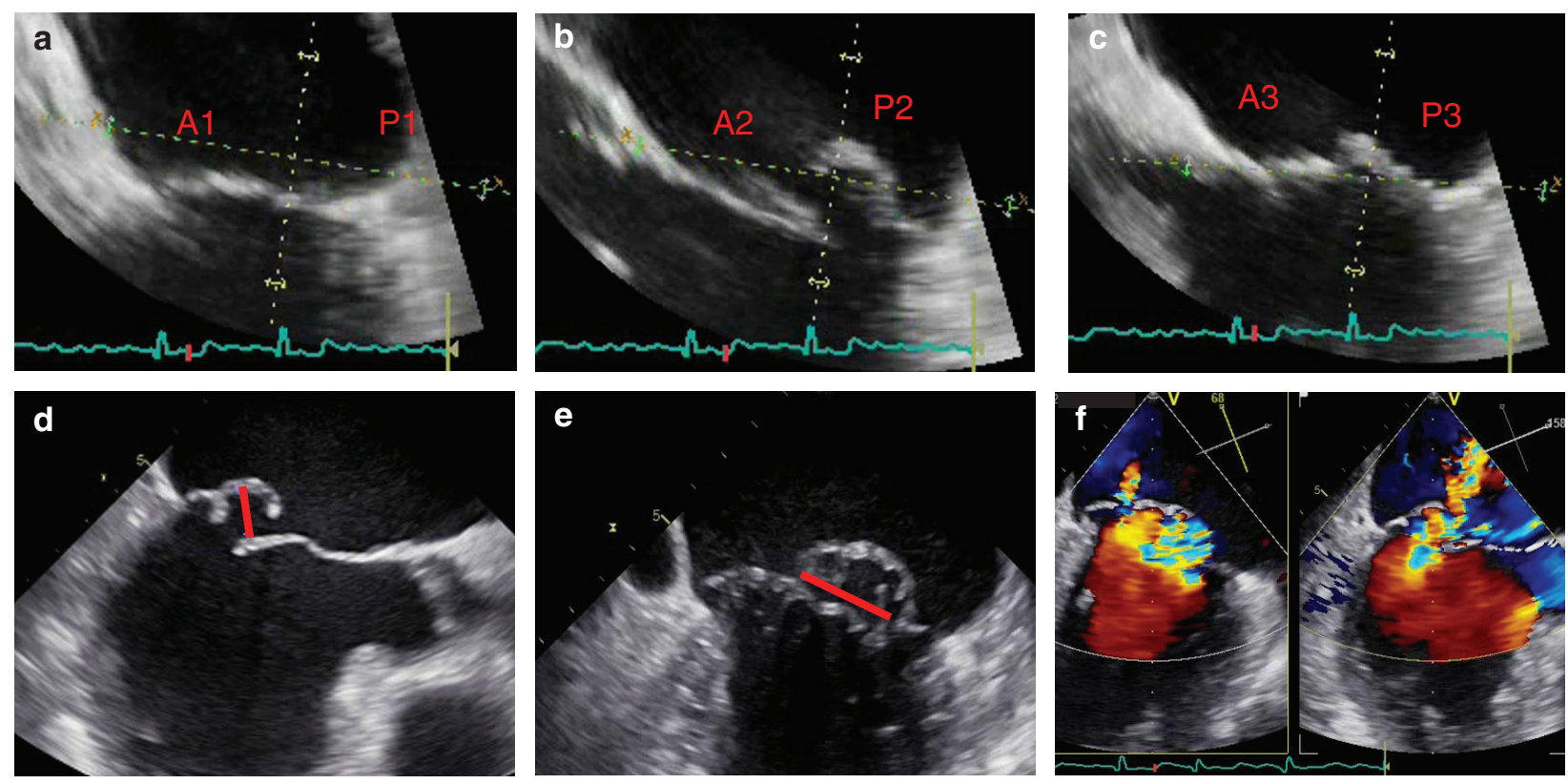

Fig. 2 a-c: Anterior and posterior mitral valve leaflet of lateral, middle, and medial position. d, e: Flail gap and width. f: Commissure and left ventricular outflow tract view on color image.

potentially promote the spread and growth of existing cancer cells. ${ }^{11)}$ On the other hand, general surgeons in the multidisciplinary team considered that the survival prognosis for this case was $>1$ year. We considered that complete cure of MR would be difficult, but acute procedure success of the MitraClip would be likely, thus reducing MR and improving symptoms of heart failure. The multidisciplinary heart team thus decided to perform the MitraClip procedure. Figure 3 shows the images of transesophageal echocardiography during MitraClip procedure. After septal puncture, we positioned and secured the first clip medially, at the A2-P2 leaflet (Fig. 3a, b). We confirmed that the length of clip insertion was $>7 \mathrm{~mm}$. Mean pressure gradient at the mitral valve was $1.5 \mathrm{mmHg}$ and moderate or over MR remained, so we decided to place a second clip lateral to the first clip. We tried to place the second clip at the site of greatest prolapse of the A2-P2 leaflets, but the actual placement site ended up being more radially situated (Fig. 3c, d). After placement of the second clip, regurgitation was reduced from severe to mild, and mean pressure gradient at the mitral valve was $2 \mathrm{mmHg}$. However, the second clip was relatively far from the first clip and resulted in triple orifices of the mitral valve. We then tried to replace the second clip closer to the first clip, to consider double orifices as standard morphology for the majority of MitraClip procedure. However, moving the second clip resulted in interference with the chorda tendineae and loss of control of the clip, with the clips becoming entangled. Figure 4 shows the results of hemodynamic monitoring and fluoroscopy at that time (Fig. $\mathbf{4 b}, \mathbf{d})$. Ventricular tachycardia occurred due to both clips touching the ventricular wall. Blood pressure fell, and hemodynamics collapsed. We attempted to bail out by trying to invert and advance the second clip to the left ventricle, then retrieved it inside the left atrium (Fig. 3e). After clip entanglement, we first suspected single leaflet device attachment because of increased mobility of the first clip and worsened regurgitation inside the first clip (Fig. 3f). However, we finally judged that single leaflet device attachment was not present because transesophageal echocardiography revealed preserved grasping of the posterior mitral leaflet. To stabilize the first clip, decrease regurgitation, and avoid entangling clips, we decided to deliver the second clip slightly more radially. Extreme care was taken in attempting delivery of the second clip, targeting more medially than the first try, and more laterally than the second try (Fig. 3g). Although MR remained medial to the first clip, severity of regurgitation was mild and acute procedure success was achieved. Finally, we confirmed that the first and second clips grasped anterior and posterior leaflets (Fig. 3h, i). Compared to the pre-procedural data, the 7-mmHg V wave disappeared and cardiac output increased. After the MitraClip procedure, symptoms of heart failure improved and he was treated on an outpatient basis. Cardiomegaly improved and pulmonary 

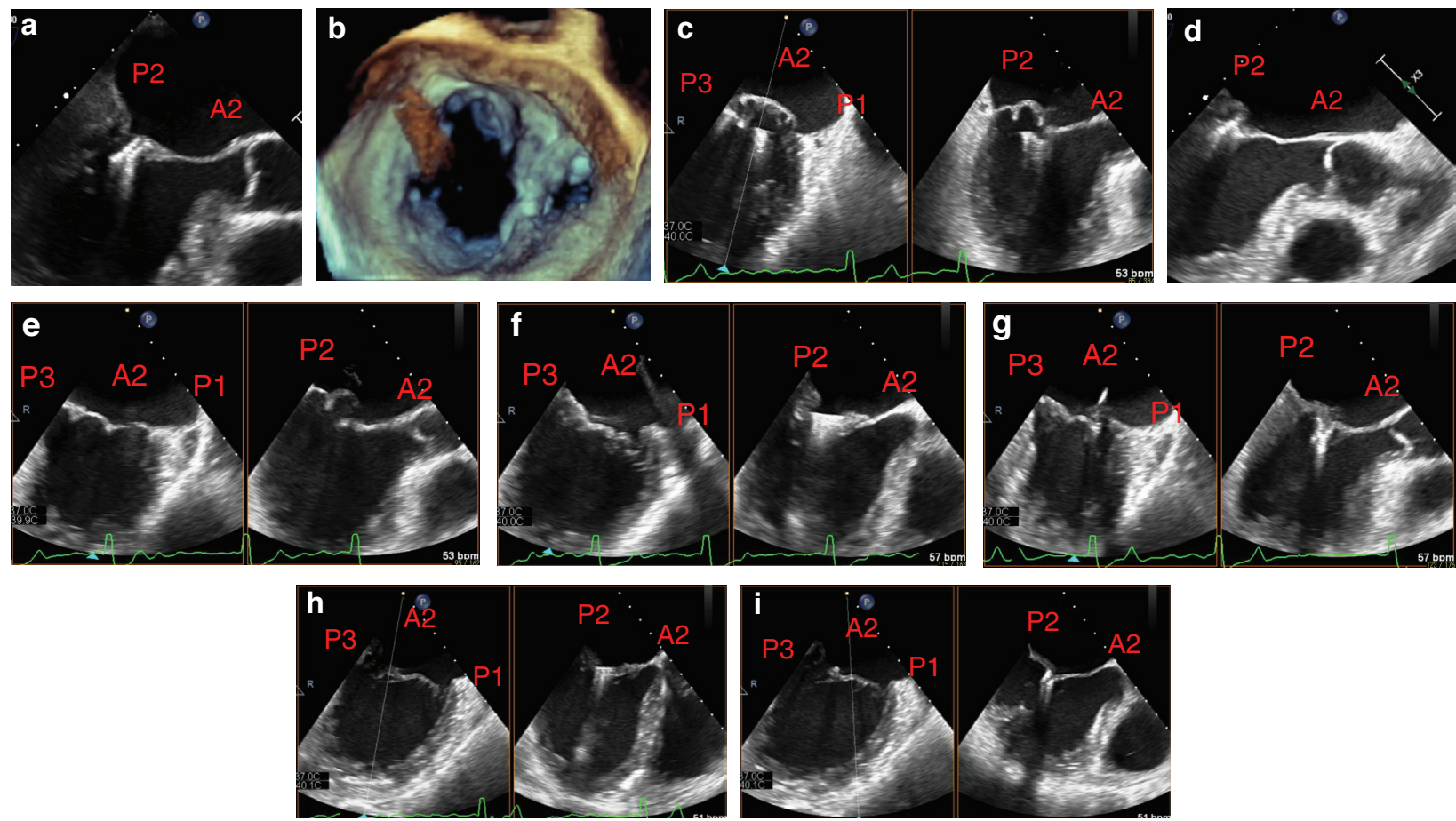

Fig. 3 The images of transesophageal echocardiography during MitraClip procedure. a, b: The detained first clip at medial position of A2-P2 leaflets. c: Greatest prolapse of the A2-P2 leaflets and located second clip. d: The placed second clip at first time. e: Leaflet and the retrieved second clip inside left atrium after clips entanglement. f: The first clip after entanglement, this finding was looked like single leaflet device attachment. g: The placed second clip at second time. $\mathbf{h}, \mathbf{i}$ : The first and second clips of final findings after detention.

blood vessel shadows were reduced on radiographic examinations (Fig. 1b).

However, X-ray examination at a regular hospital visit 5 months after the procedure showed a change in the angle between the two clips (Fig. 1c). Transthoracic echocardiography showed detachment of the first clip from the posterior leaflet, with attachment only to the anterior leaflet (Fig. 1g). The first clip thus appeared to show single leaflet device attachment. Options of performing surgery or a second attempt at the MitraClip procedure was therefore considered. At 6 months after the procedure, while considering these options, the patient showed exacerbation of dyspnea with light exertion. Chest X-ray showed a detached clip in the left ventricle (Fig. 1d). Transesophageal echocardiography showed worsened MR and absence of the first (medialside) clip (Fig. 1h). The clip that had dropped out was now attached to the inferior wall of the left ventricular apex. We diagnosed embolization of the first clip, and emergent surgery was performed. Replacement of the mitral valve with a mechanical valve was performed and the left ventricular clip was removed (Fig. 5). Severe mucoid degeneration of both leaflets was observed, particularly of the posterior leaflet, and the chordae tendineae were torn, suggesting a Barlow-type valve morphology. The second clip was seen firmly grasping the anterior and posterior leaflets (Fig. 5a, b). However, the medial-side clip (first clip) had been dislodged from the mitral valve. The clip removed from the left ventricle had stayed lodged in the apical left ventricular myocardium. Valve tissue was identified in the grasping area of the embolized clip, and we considered that the anterior leaflet had been torn after single leaflet device attachment. There was a site suggesting a torn mark on the anterior leaflet (Fig. 5a, red arrow), but there was no such mark findings on the posterior leaflet. From the clinical course of echocardiography, the posterior leaflet came off and became single leaflet device attachment, which was the grip of only the anterior leaflet, followed by clip embolism. Although it was inferred, it was thought that the anterior leaflet held by the clip by single leaflet device attachment was torn off and a clip embolism was caused. No intraoperative complications were encountered and the postoperative course was good. 

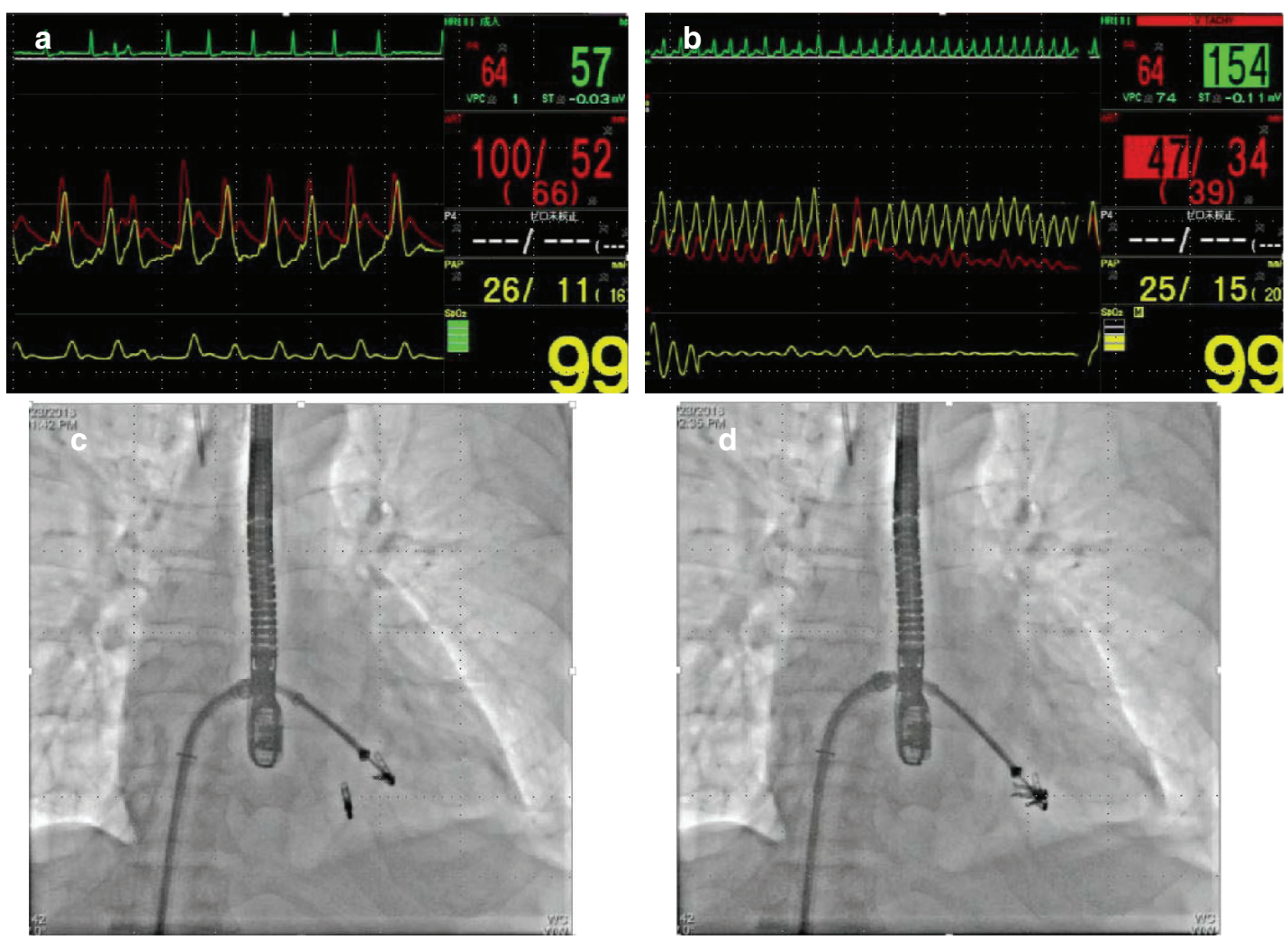

Fig. 4 a, c: The hemodynamic display screen and fluoroscopic image on insertion of the second clip in the MitraClip procedure. b, d: The hemodynamic display screen and fluoroscopic image on clip entanglement, showing ventricular tachycardia and deteriorating hemodynamics.

No progression of pancreatic cancer was identified postoperatively.

\section{Discussion}

Single leaflet device attachment of a MitraClip has been reported in $0.7-6.3 \%$ of cases, and device embolization in $0.3 \%$ of cases. ${ }^{3,4,8,9)}$ Device embolization is rare, with most previous reports describing MitraClip embolization during clip deployment. ${ }^{12-16)}$ Rate of single leaflet device attachment were $6.3 \%$ and $0.7 \%$ in EVELEST trial for degenerative MR and COAPT trial for functional MR. ${ }^{3,5)}$ Although a simple comparison is not possible, single leaflet device attachment was lower rate for functional MR compared to degenerative MR. Additionally, single leaflet device attachment of MitraClip has decreased compared to the initial date, and a learning curve is important. ${ }^{5,7)}$ Late embolization of the MitraClip after deployment appears extremely rare. In one previous report, myocardial infarction due to MitraClip embolization occurred 6 weeks after MitraClip deployment. ${ }^{12)}$ In another case, embolization of a MitraClip into the right axillary artery occurred 1 year after clip deployment. ${ }^{13)}$ In a third case, a MitraClip embolized to the renal artery. ${ }^{14)}$ In the previous reports, all late embolism occurred with functional MR. In the previous reports, all late embolism occurred with functional MR. In our case, however, embolization occurred with degenerative MR, and furthermore this is the first case of the clips entangled during the MitraClip procedure. We somehow bailed out from entanglement of two clips and retrieved inside left atrium, but it appears that echocardiography was not enough to confirm the stability of clip. The severity of MR was mild and we decided that acute procedure success was achieved. True procedural success in this case should have consisted of not only reduction of MR but also clip stability, given the background of severe degenerative MR. Therefore, we might have to implant the additional clip to obtain the clip stability, or we should consider open heart surgery at that time.

In addition, during follow-up period, when the clip became more instable and single leaflet device attachment 

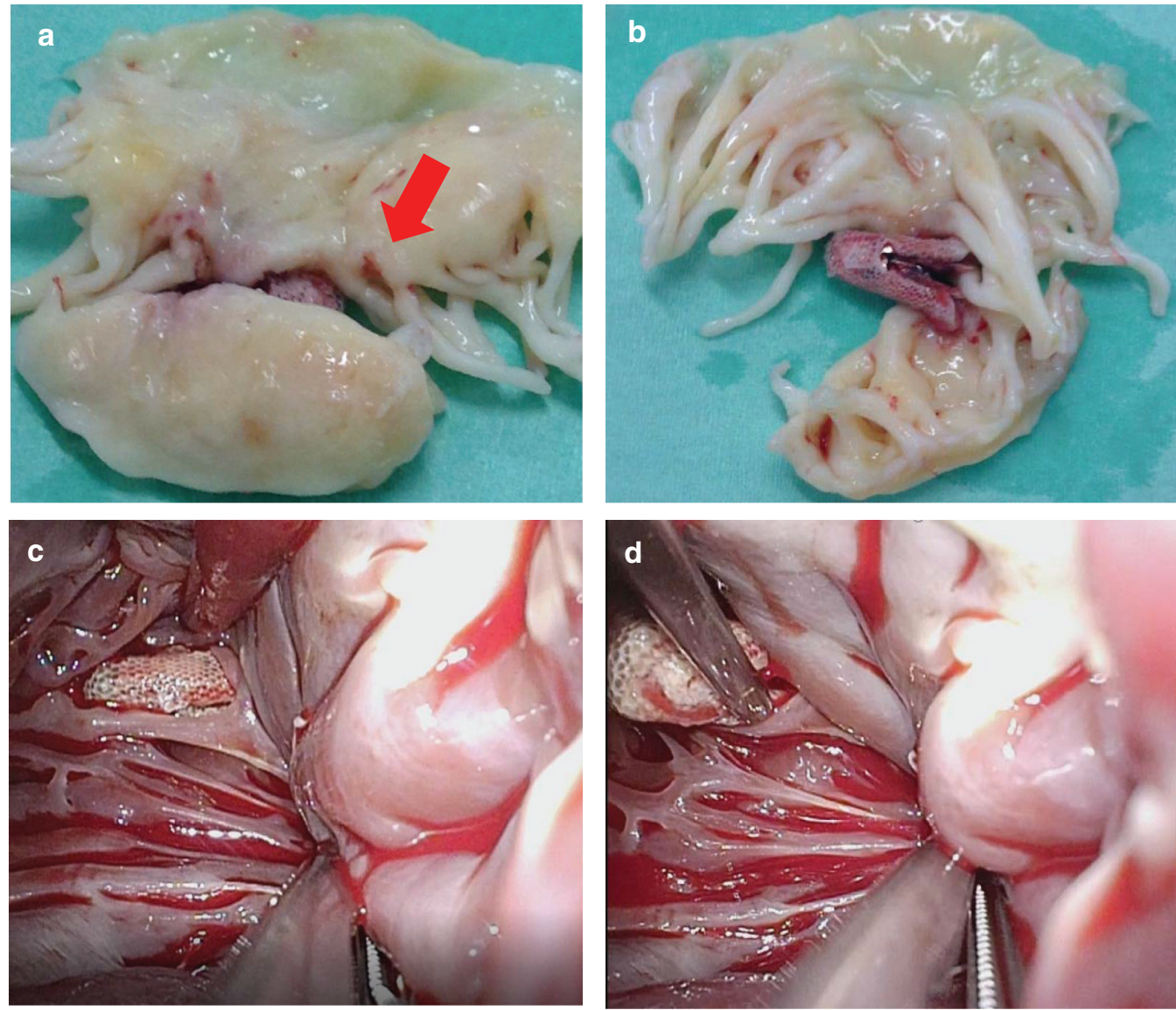

Fig. 5 The second clip firmly attached to the mitral valve resected during open heart surgery, and the detached first clip recovered in the surgical operation. a: The resected mitral valve and second clip. b: Viewed from the left ventricular side. c: The first clip in left ventricle. d: The first clip removed from the left ventricle.

was highly suspected, we should have decided to perform open heart surgery immediately.

Wide flail of the posterior mitral leaflet, as in Barlow syndrome, led to difficult deployment of the MitraClip device in this case. Barlow syndrome is classified as a morphological contraindication for the MitraClip procedure by the German consensus criteria, ${ }^{10)}$ but we decided to perform the MitraClip because of history of advanced cancer. There has been only one previous report that MitralClip procedure was performed for Barlow disease. ${ }^{17)}$ In that report, MitraClip procedure was successfully treated by implanting MitraClip XTR device. MitraClip XTR device, which features longer arms for grasping and grasping width is $5 \mathrm{~mm}$ longer than NT divece. ${ }^{18)}$ MitraClip XTR system is recommended for patients with large flail, as in Barlow syndrome. ${ }^{18)}$ Since XTR device is not yet approved in Japan, it may be necessary to add more clips than usual to increase the grasping area and stability of clips. Con- sidering that single leaflet device attachment is decreasing due to experience and Barlow disease is an exclusion criterion in EVELEST trials, it was thought that MitraClip procedure should not be performed for Barlow disease, especially in facilities where experience is not sufficient and in situations where only MitraClip Nt device can be used.

We created three orifices after the second clip implantation after the first try. MR jet was reduced to mild without mitral stenosis. Creating triple orifices technique may be associated with an improved long-term prognosis, ${ }^{19)}$ until the benefit becomes established, we consider that this technique should only be applied for a limited morphology in whom available grasping position of mitral leaflet is limited especially by calcified leaflet.

The experience of this case suggests three key points. First, clip instability may carry high risks of single leaflet device attachment and clip embolization in the 
MitraClip procedure. Second, the progression of clip instability and leaflet device attachment in follow-up period warrants immediate treatment. Final, although there is a tradeoff with risk, adaptation to Barlow disease should be carefully considered in the MitraClip NT system.

\section{Conclusion}

In MitraClip procedure, not only reduction of MR but also clip stability is important factors to determine success of the MitraClip procedure, particularly in cases with degenerative MR.

\section{Author contributions}

Research concept and design: Nobuyuki Fukuda, Hiroshi Ueno, Shuhei Tanaka, Ryuichi Ushijima, and Mitsuo Sobajima.

Collection and analysis of research data: Nobuyuki Fukuda, Hiroshi Ueno, Shuhei Tanaka, Ryuichi Ushijima, and Mitsuo Sobajima.

Drafting manuscript: Nobuyuki Fukuda, Hiroshi Ueno, Shuhei Tanaka, Ryuichi Ushijima, and Mitsuo Sobajima.

Interpretations of research data and critical recommendations on important intellectual content of manuscript: Nobuyuki Fukuda, Hiroshi Ueno, Shuhei Tanaka, Ryuichi Ushijima, and Mitsuo Sobajima.

Final approval of submitted papers and published manuscripts: all authors

Consent to accountability for research: all authors

\section{Disclosure Statement}

The first author and co-authors have no conflicts of interest to declare.

\section{References}

1) Nishimura RA, Otto CM, Bonaw RO, et al: 2017 AHA/ACC focused update of the 2014 AHA/ACC Guideline for the management of patients with valvular heart disease: a report of the American College of Cardiology/American Heart Association task force on clinical practice Guidelines. J Am Coll Cardiol 2017; 70: 252-289.

2) Baumgartner H, Falk V, Bax JJ, et al: $2017 \mathrm{ESC} /$ EACTS guidelines for the management of valvular heart disease. Eur Heart J 2017; 38: 2739-2791.
3) Stone GW, Lindenfeld J, Abraham WT, et al: Transcatheter mitral-valve repair in patients with heart failure. N Engl J Med 2018; 379: 2307-2318.

4) Feldman T, Wasserman HS, Herrmann HC, et al: Percutaneous mitral valve repair using the edge-toedge technique: six-month results of the EVEREST Phase I Clinical Trial. J Am Coll Cardiol 2005; 46: 2134-2140.

5) Feldman $T$, Kar $S$, Rinaldi $M$, et al: Percutaneous mitral repair with the MitraClip system: safety and midterm durability in the initial EVEREST (Endovascular Valve Edge-to-Edge REpair Study) cohort. $J$ Am Coll Cardiol 2009; 54: 686-694.

6) Franzen O, van der Heyden J, Baldus S, et al: MitraClip ${ }^{\circledR}$ therapy in patients with end-stage systolic heart failure. Eur J Heart Fail 2011; 13: 569-576.

7) Feldman T, Foster E, Glower DD, et al: Percutaneous repair or surgery for mitral regurgitation. $N$ Engl $J$ Med 2011; 364: 1395-1406.

8) Whitlow PL, Feldman T, Pedersen WR, et al: Acute and 12-month results with catheter-based mitral valve leaflet repair: the EVEREST II (Endovascular Valve Edge-to-Edge Repair) high risk study. J Am Coll Cardiol 2012; 59: 130-139.

9) Maisano F, Franzen O, Baldus S, et al: Percutaneous mitral valve interventions in the real world: Early and 1-year results from the ACCESS-EU, a prospective, multicenter, nonrandomized post-approval study of the MitraClip therapy in Europe. J Am Coll Cardio 2013; 62: 1052-1061.

10) Boekstegers P, Hausleiter J, Baldus S, et al: Percutaneous interventional mitral regurgitation treatment using the Mitra-Clip system. Clin Res Cardiol 2014; 103: 85-96.

11) Pinto CA, Marcella S, August DA, et al: Cardiopulmonary bypass has a modest association with cancer progression: a retrospective cohort study. BMC Cancer 2013; 13: 519.

12) Chitsaz S, Jumean M, Dayah T, et al: Late MitraClip embolization: a new cause of ST-segment-elevation myocardial infarction. Circ Cardiovasc Interv 2016; 9: $\mathrm{e} 004271$.

13) Ak Sivrikoz İ, Önner H, Kasapoğlu Dündar E, et al: F-18 FDG PET/CT images of a rare primer cardiac tumour: primary pericardial mesothelioma. Anatol $J$ Cardiol 2016; 16: 635-636.

14) Alozie A, Westphal B, Kische S, et al: Surgical revision after percutaneous mitral valve repair by edge-to-edge device: when the strategy fails in the highest risk surgical population. Eur J Cardiothorac Surg 2014; 46: 55-60.

15) Paranskaya L, D’Ancona G, Bozdag-Turan I, et al: Early and mid-term outcomes of percutaneous mitral valve repair with the MitraClip ${ }^{\circledR}$ : comparative analysis of different EuroSCORE strata. EuroIntervention 2012; 8: 571-578. 
16) Seeburger J, Raschpichler M, Lurz P, et al: Late device embolization after transcatheter mitral valve edge-to-edge repair. Eur Heart J 2017; 38: 1260 .

17) Weinmann K, Markovic S, Rottbauer W, et al: First experience with the MitraClip XTR device for extensive mitral valve prolapse (Barlow's disease). EuroIntervention 2018; 14: e1276-e1277.
18) Praz F, Braun D, Unterhuber M, et al: Edge-to-edge mitral valve repair with extended clip arms: early experience from a multicenter observational study. JACC Cardiovasc Interv 2019; 12: 1356-1365.

19) Paranskaya L, Kische S, Bozdag-Turan I, et al: Mitral valve with three orifice after percutaneous repair with the MitraClip system: the triple-orifice technique. Clin Res Cardiol 2012; 101: 847-849. 